\title{
The Prevalence and Covariates of Potential Doping Behavior in Kickboxing; Analysis Among High-Level Athletes
}

\author{
by \\ Damir Sekulic ${ }^{1}$, Natasa Zenic ${ }^{1}$, Sime Versic ${ }^{2}$, Dora Maric ${ }^{3}$, Goran Gabrilo ${ }^{1}$, \\ Mario Jelicic ${ }^{1}$
}

\begin{abstract}
The official reports on doping behavior in kickboxing are alarming, but there have been no empirical studies that examined this problem directly. The aim of this study was to investigate the prevalence, gender differences and covariates of potential-doping-behavior, in kickboxing athletes. A total of 130 high-level kickboxing athletes (92 males, $21.37 \pm 4.83$ years of age, $8.39 \pm 5.73$ years of training experience; 38 women, $20.31 \pm 2.94$ years of age; $9.84 \pm 4.74$ years of training experience) completed questionnaires to study covariates and potential-doping behavior. The covariates were: sport factors (i.e. experience, success), doping-related factors (i.e. opinion about penalties for doping users, number of doping testing, potential-doping-behavior, etc.), sociodemographic variables, task-and ego-motivation, knowledge on sports nutrition, and knowledge on doping. Gender-based differences were established by independent ttests, and the Mann-Whitney test. Multinomial logistic regression analyses were performed to define the relationships between covariates and a tendency toward potential-doping behavior (positive tendency - neutral - negative tendency). The potential-doping behavior was higher in those athletes who perceived kickboxing as doping contaminated sport. The more experienced kickboxers were associated with positive intention toward potential-doping behavior. Positive intention toward potential-doping behavior was lower in those who had better knowledge on sports nutrition. The taskand ego-motivation were not associated to potential-doping behavior. Because of the high potential-doping-behavior (less than $50 \%$ of athletes showed a negative tendency toward doping), and similar prevalence of potential-doping behavior between genders, this study highlights the necessity of a systematic anti-doping campaign in kickboxing. Future studies should investigate motivational variables as being potentially related to doping behavior in younger kickboxers.
\end{abstract}

Key words: performance enhancing drugs, martial arts, task and ego motivation.

\section{Introduction}

Kickboxing is a stand-up martial art that allows both punching and kicking and includes techniques from different combat sports (Nassib et al., 2011). Kickboxing competitions are organized by weight categories and athletes frequently engage in voluntary rapid weight loss (weight-cutting) (Franchini et al., 2012; Pettersson et al., 2013). With a rate of 40 injuries per $1000 \mathrm{~min}$ of practice, the risk of injury in kickboxing is high
(Lystad, 2015). As in other sports with a high prevalence of injury, in which athletes experience pain and are engaged in weight-cutting, the consumption of performance enhancers, including those that are prohibited by the World AntiDoping Agency (WADA) is expected to be high (Babwah, 2014). However, to the best of our knowledge, no studies have directly investigated this problem in kickboxing. In the meantime, the

1 - University of Split, Faculty of Kinesiology, Split, Croatia.

2 - University of Split, University Sports Federation, Split, Croatia.

3 - Taekwondo Club Energy, Split, Croatia. 
WADA has reported disturbing figures on potential violations of anti-doping rules in this sport (WADA, 2015).

Sports authorities are highly interested in evidencing factors that could be related to doping behavior in athletes for its potential to contribute to anti-doping efforts, with the main idea: (i) to control risk-factors of doping behavior, and/or (ii) to proclaim and encourage those factors evidenced as protective against doping (Sekulic et al., 2014). In a study conducted on internationallevel tennis players, Kondric et al. (2013) reported knowledge on sports nutrition as a protective factor of potential doping behavior. Nutritional supplementation is found to be associated with increased likelihood of doping behavior (Backhouse et al., 2013; Sekulic et al., 2014). Sport specific variables such as sport-achievement and type of sport (individual vs. team-sport) are differentially associated to potential and current doping behavior (Rodek et al., 2012, 2013). While most of the studies showed sport-specific, and socio-culturally specific associations between studied factors and doping behavior, there is a general consensus that the personal belief of higher doping occurrence in sport is strongly associated with higher doping likelihood (Kondric et al., 2011; Rodek et al., 2013; Sekulic et al., 2016). However, there is an evident lack of knowledge on factors specifically related to doping behavior in martial arts.

The main energizing force in sport is an athlete's need to demonstrate his/hers sport competence (i.e. sports performance) (Baric and Bucik, 2009). According to the Achievement Goal Theory, the need for competence is frequently realized throughout the sport competition and self-perception of ability becomes the central variable (Baric and Horga, 2007). The athletes' self-perception of ability is directly modulated by sport achievement (i.e. sport result), and their motivation is generally considered to be one of the most important factors that shape conditions and prerequisites for success (Baric and Bucik, 2009). Since doping in sport is used to improve performance and consequently to achieve success, it is reasonable to expect that certain relationships between motivation and doping behavior exist. However, only few studies actually reported associations between motivational factors and doping behavior in athletes (Allen et al., 2015; Sas-
Nowosielski and Swiatkowska, 2008; Zucchetti et al., 2015).

When studying Polish athletes, the authors reported ego orientation as being negatively, while task orientation as being positively related to attitudes toward doping (SasNowosielski and Swiatkowska, 2008). While studying psychological and social correlates of doping attitudes in Italian athletes, other authors highlighted extrinsic motivation as predictor of positive attitude toward doping (Zucchetti et al., 2015). Finally, task and ego goals along with the mastery motivational climate were recently found to be predictors of attitudes to performanceenhancing drugs in elite Scottish athletes (Allen et al., 2015). To the best of our knowledge, there is no study that has examined the motivational factors as being potentially related to doping behavior in martial arts, including kickboxing.

This study aimed to investigate the prevalence of potential doping behavior, and the factors associated with doping behavior in high level kickboxing athletes. More specifically, we examined (i) potential doping behavior in kickboxing, (ii) gender-differences in potential doping behavior and associated factors, and (iii) sport-related-factors, socio-demographic variables, knowledge on sports nutrition and doping, task- and ego-orientation, as covariates of potential doping behavior in high level kickboxers. The leading hypotheses of the study were: (i) significant differences in potential doping behavior and other studied factors (age, knowledge on sports nutrition and doping, taskand ego-orientation, educational level, kickboxing experience, competitive-weight category, competitive-achievement, consumption of dietary supplements, number of doping testing, opinion on doping penalties, opinion on main problem of doping, and opinion on occurrence of doping in kickboxing) would be evidenced between genders, and (ii) studied covariates (i.e. gender and previously specified factors) would be associated to potential doping behavior.

\section{Methods}

\section{Participants}

In this cross-sectional study, the participants were 130 kickboxers (92 males, 21.37 \pm 4.83 years of age, $8.39 \pm 5.73$ years of training experience; 38 women, $20.31 \pm 2.94$ years of age; 
$9.84 \pm 4.74$ years of training experience) from five countries. All the participants were tested in 2014 and early 2015.

\section{Measures}

The variables were collected via four structured questionnaires: the questionnaire on substance use (QSU) (Kondric et al., 2011; Sekulic et al., 2008), the knowledge of sports nutrition questionnaire $(\mathrm{KSN})$, the knowledge of doping questionnaire (KD) (Sekulic et al., 2014) and the Croatian version of the task and ego orientation in sport questionnaire (CTEOSQ) (Baric and Horga, 2007). The QSU assessed athletes' sociodemographic data as well as sport-, sports nutrition- and doping-related factors. The sociodemographic data included age, gender and educational level achieved. Sport factors consisted of questions on (i) weight category (later divided into: low-, middle- and heavy-weight), (ii) experience in kickboxing (years), and (iii) the highest competitive result achieved (three itemscale: national medal - international Medal - top ranked international tournament medal). Sports nutrition and doping factors were assessed by asking participants about (i) their consumption of dietary supplements, (ii) their opinions on doping occurrence in kickboxing, (iii) their potential doping behavior, (iv) the number of times they had undergone doping testing, (v) their personal opinions on the main problem with doping, and (vi) their personal opinions on the penalties for doping offenses. Within the QSU questionnaire athletes responded on the question about his/her potential doping behavior (responses included: "I will use doping if it helps me", "Not sure", "I do not intend to use doping in future") which was later used as a multinomial criterion variable (see later text on statistics). The KD questionnaire consisted of 10 questions. Athletes were asked about doping side effects (i.e. "The use of amphetamines by women is related to male-like changes in body appearance"), and doping regulation issues (i.e. "If an athlete has an out-ofcompetition doping test, four weeks should elapse before their next doping test"). Each question (statement) was in a "true or false" format; if the answer was correct, the athletes scored one point. The final results ranged from 0 to 10 (Sekulic et al., 2014). The KSN consisted of test questions using the same evaluation system as previously explained for the KD. Three items examined knowledge of hydration/dehydration (i.e. "The negative side effects of excessive sweating are best cured by drinking pure water"), and knowledge of nutrition strategies aimed at recovery (i.e. "Protein supplementation requires an increased intake of water"), while four items targeted general knowledge of nutrition (i.e. "Fresh fruit and vegetables are the best source of high-quality proteins") (Sekulic et al., 2014). The CTEOSQ consisted of 13 questions evidencing intrinsic (7items; i.e. "I would like to continue practicing like that"), and extrinsic- (6-items; i.e. "I'm able to execute the task better than my peers") motivation of the subjects. The participants responded on a Likert scale of 5 points (ranging from strongly disagree to strongly agree) (Baric and Horga, 2007).

\section{Procedures}

Testing was arranged in groups of at least three athletes, who were informed that the survey was strictly anonymous, that they could withdraw from the study at any time, that they could leave some of the questions and/or the entire questionnaire unanswered, and that returning the completed questionnaire would be considered consent to participate in the study. All of the athletes answered the questionnaire in similar conditions, and one of the investigators was available on demand for possible questions and explanations. Approximately 10-12 min were necessary to fulfill the questionnaire form. After answering, athletes placed the questionnaire in the envelope, sealed it and placed it in a closed box. The box was opened the day after testing by the examiner who did not test the participants. The response rate was higher than $99 \%$, and only one athlete returned the questionnaire unanswered. The study complied with all ethical guidelines and received the approval of the Institutional Ethical Board at the corresponding author's institution (Ethical Board Approval No 10/09/2014-1). For the purposes of this study, reliability of the questionnaires was tested in 12 athletes who responded to a questionnaire twice within two weeks. The percentage of equally answered statements in the QSU was more than $90 \%$, with a test-retest correlation of 0.89 for the $\mathrm{KD}$ and of 0.84 for the $\mathrm{KSN}$, demonstrating appropriate reliability of the measuring tool. The reliability of the CTEOSQ was checked separately for the task- and ego-subscale and was also found 
to be high (test-retest correlation of 0.91 and 0.93 , respectively). The Cronbach Alpha values calculated for the total sample were 0.89 (task-) and 0.91 (ego-subscale).

\section{Statistical Analysis}

The Kolmogorov Smirnov (KS) test evidenced variables derived from the CTEOSQ (task- and ego-orientation), KSN and KD, experience in kickboxing and age, as normally distributed (d value: $0.03,0.04,0.06,0.08,0.10$, 0.09 ; all $p>0.05$, respectively), and for these variables means and standard deviations are reported. Meanwhile, other variables derived from the QSU were identified as nonparametric (Kolmogorov Smirnov's d values $>0.20$; all $p<$ 0.05). Therefore, counts (frequencies) and percentages were calculated for all QSU variables, but age and experience in kickboxing. The differences between genders were established by the Mann-Whitney test (for non-parametric variables such as "Educational level", "Competitive achievement"), and independent samples t-tests (for parametric variables, such as $\mathrm{KSN}, \mathrm{KD}$, task- and ego-orientation). Multinomial logistic regression models were employed to examine how variables derived by the QSU, KSN, $\mathrm{KD}$ and CTEOSQ were associated with the potential doping behavior (Negative - Not sure Positive). The negative self-declared doping tendency was set as reference value. While previous studies identified significant associations between personal belief about doping presence in sport and doping-behavior, the logistic analyses calculations consisted of two phases. In the first phase we calculated multinomial logistic regression between "personal opinion about doping presence in kickboxing" as a covariate of "potential doping behavior". Since results showed a strong relationship between these two variables (see Results for more details), in the second phase we calculated relationships between remaining covariates and criterion, by controlling "personal opinion about doping presence in kickboxing" as a confounding variable. For all analyses, a $p$-level of $95 \%$ was applied.

\section{Results}

There were no significant gender differences in age, experience in kickboxing, $\mathrm{KD}$ and ego-orientation. Females were more strongly task-oriented, and achieved higher scores in the KSN than males (Table 1)

Table 1

Descriptive statistics and differences between genders for parametric variables

\begin{tabular}{|c|c|c|c|c|c|c|}
\hline & \multicolumn{2}{|c|}{ Males } & \multicolumn{2}{|c|}{ Females } & \multicolumn{2}{|c|}{ T-TEST } \\
\hline & Mean & $\mathrm{SD}$ & Mean & $\mathrm{SD}$ & T-value & $p$ \\
\hline AGE (years) QSU & 21.37 & 4.83 & 20.31 & 2.94 & 1.25 & 0.21 \\
\hline EXPERIENCE IN KICKBOXING (years) QSU & 8.39 & 5.73 & 9.84 & 4.74 & -1.37 & 0.17 \\
\hline KNOWLEDGE ON DOPING (score) KD & 3.16 & 1.11 & 3.01 & 1.02 & 1.25 & 0.22 \\
\hline KNOWLEDGE ON NUTRITION (score) KSN & 5.71 & 2.75 & 6.76 & 2.27 & -4.00 & 0.01 \\
\hline TASK MOTIVATION (score) CTEOSQ & 6.74 & 0.94 & 7.41 & 1.15 & -2.00 & 0.04 \\
\hline EGO MOTIVATION (score) CTEOSQ & 5.71 & 0.65 & 5.12 & 0.57 & 1.01 & 0.41 \\
\hline
\end{tabular}

QSu presents variables derived from the Questionnaire of Substance Use;

${ }_{K D}$ presents variables derived from the Knowledge on Doping Questionnaire;

${ }_{K S N}$ presents variables derived from the Knowledge on Sport Nutrition Questionnaire;

CTEOSQ presents variables derived from the Croatian Version of the Task and Ego Orientation In Sport Questionnaire 
Table 2

Descriptive statistics ( $F$ - frequency, \% - percentage) and differences between genders (Mann Whitney test - MW) for nonparametric variables obtained by the Questionnaire of Substance Use

\begin{tabular}{|c|c|c|c|c|}
\hline & \multirow{2}{*}{$\begin{array}{c}\text { Males } \\
\mathrm{F}(\%)\end{array}$} & \multirow{2}{*}{$\begin{array}{c}\text { Females } \\
\text { F (\%) }\end{array}$} & \multicolumn{2}{|c|}{ MW } \\
\hline & & & $\mathrm{Z}$ & $p$ \\
\hline EDUCATIONAL LEVEL & & & -1.96 & 0.04 \\
\hline Elementary school & $0(0)$ & $0(0)$ & & \\
\hline High school & $66(71.7)$ & $20(52.6)$ & & \\
\hline Student & $16(17.4)$ & $12(31.6)$ & & \\
\hline College/University degree & $10(10.9)$ & $6(15.8)$ & & \\
\hline KICKBOXING RESULT ACHIEVED & & & -2.00 & 0.04 \\
\hline Participation in National Championships & $12(13)$ & $0(0)$ & & \\
\hline National Championship Medal & $10(10.9)$ & $2(5.3)$ & & \\
\hline International Tournament Medal & $44(47.8)$ & $22(57.9)$ & & \\
\hline Top Ranked International Tournament Medal & $26(28.3)$ & $14(36.8)$ & & \\
\hline DIETARY SUPPLEMENTATION & & & -3.01 & 0.01 \\
\hline Regularly & $66(71.7)$ & $16(42.1)$ & & \\
\hline From time to time & $26(28.3)$ & $22(57.9)$ & & \\
\hline Rarely or no supplementation & $0(0)$ & $0(0)$ & & \\
\hline \multicolumn{5}{|l|}{ POTENTIAL DOPING BEHAVIOR } \\
\hline I don't intend to use doping (negative) & $44(47.8)$ & $16(42.1)$ & 0.05 & 0.90 \\
\hline I'm not sure (neutral) & $26(28.3)$ & $16(42.1)$ & & \\
\hline I intend to use doping in the future if it helps me (positive) & $22(23.9)$ & $6(15.8)$ & & \\
\hline \multicolumn{5}{|l|}{ EVER TESTED ON DOPING * } \\
\hline No & $76(82.6)$ & $30(79.0)$ & & \\
\hline Yes & $16(17.4)$ & $8(21.0)$ & & \\
\hline \multicolumn{5}{|l|}{ DOPING IN KICKBOXING * } \\
\hline I don't think that doping occurs & $4(4.4)$ & $0(0)$ & & \\
\hline Not sure of doping in kickboxing & $24(26.1)$ & $18(47.4)$ & & \\
\hline Occurs, but rarely & 18 (19.6) & $12(31.6)$ & & \\
\hline Doping is common & $46(50.0)$ & $8(21.1)$ & & \\
\hline \multicolumn{5}{|l|}{ MAIN PROBLEM OF DOPING * } \\
\hline Doping is mainly a health-hazard & $44(48.1)$ & $19(50.0)$ & & \\
\hline Doping is mainly a problem of fair-play & $48(51.9)$ & $19(50.0)$ & & \\
\hline DOPING PENALTIES & & & -0.44 & 0.65 \\
\hline Lifelong suspension & $44(48.1)$ & $18(48.4)$ & & \\
\hline Suspension for a couple of seasons & $38(41.3)$ & $16(42.1)$ & & \\
\hline Financial punishment/Doping should be allowed & $10(10.9)$ & $4(10.5)$ & & \\
\hline
\end{tabular}

* Mann Whitney test was not performed (nominal variables) 
Table 3

Multinominal logistic regression estimates of covariates for potential doping behavior

\begin{tabular}{|c|c|c|c|c|}
\hline & \multirow{3}{*}{$\begin{array}{c}\text { Nagelkerke's } \\
\text { R square }\end{array}$} & \multicolumn{3}{|c|}{ Potential doping behavior } \\
\hline & & Positive attitude & Not sure & \multirow{2}{*}{$\begin{array}{l}\text { Negative } \\
\text { attitude }\end{array}$} \\
\hline & & AOR $(95 \% C I)$ & AOR $(95 \% C I)$ & \\
\hline AGE (continuous) QSU & 0.05 & $1.14(0.91-1.19)$ & $1.18(0.94-1.36)$ & REF \\
\hline EXPERIENCE IN KICKBOXING (continuous) QSU & 0.20 & $1.11(1.00-1.23) *$ & $1.10(0.99-1.23)$ & REF \\
\hline KNOWLEDGE ON NUTRITION (continuous) KSN & 0.24 & $0.62(0.33-0.98) *$ & $0.66(0.35-1.25)$ & REF \\
\hline KNOWLEDGE ON DOPING (continuous) KD & 0.02 & $0.94(0.58-1.54)$ & $1.30(0.76-2.14)$ & REF \\
\hline TASK ORIENTATION (continuous) CTEOSQ & 0.05 & $0.87(0.11-2.12)$ & $0.98(0.41-4.54)$ & REF \\
\hline EGO ORIENTATION (continuous) CTEOSQ & 0.07 & $1.00(0.41-5.98)$ & $0.98(0.66-4.98)$ & REF \\
\hline GENDER QSU & 0.02 & & & \\
\hline Male & & $0.75(0.26-2.18)$ & $0.44(0.14-1.32)$ & \\
\hline Female & & REF & REF & REF \\
\hline COMPETITIVE WEIGHT CATEGORY QSU & 0.06 & & & \\
\hline Low weight & & $0.69(0.19-2.43)$ & $0.74(0.20-2.74)$ & \\
\hline Middle weight & & $0.90(0.33-2.46)$ & $0.74(0.25-2.17)$ & \\
\hline Heavy weight & & REF & REF & REF \\
\hline EDUCATION QSU & 0.05 & & & \\
\hline High school & & $0.73(0.14-3.96)$ & $0.28(0.05-1.45)$ & \\
\hline Student & & $0.56(0.08-3.69)$ & $0.50(0.08-3.13)$ & \\
\hline College/University degree & & REF & REF & REF \\
\hline COMPETITIVE ACHIEVEMENT QSU & 0.09 & & & \\
\hline National Championship Medal & & $0.45(0.09-2.14)$ & $0.21(0.03-1.51)$ & \\
\hline International Tournament Medal & & $0.43(0.15-1.29)$ & $0.52(0.17-1.64)$ & \\
\hline Top Ranked International Tournament Medal & & REF & REF & REF \\
\hline DIETARY SUPPLEMENTATION QSU & 0.19 & & & \\
\hline Regularly & & REF & REF & \\
\hline From time to time & & $2.30(0.92-58.75)$ & $4.27(1.52-11.97)^{*}$ & REF \\
\hline \multicolumn{5}{|l|}{ DOPING PENALTIES QSU } \\
\hline Lifelong suspension & & $0.78(0.11-1.99)$ & $0.54(0.11-8.98)$ & \\
\hline Suspension for a couple of seasons & & $0.89(0.21-7.87)$ & $0.55(0.11-9.11)$ & \\
\hline Financial punishment/doping should be & & REF & REF & REF \\
\hline EVER TESTED ON DOPING QSU & 0.08 & & & \\
\hline No & & $1.14(0.55-2.41)$ & $1.42(0.67-2.98)$ & \\
\hline Yes & & REF & REF & REF \\
\hline THE MAIN PROBLEM OF DOPING QSU & 0.06 & & & \\
\hline Doping is mainly a health-hazard & & REF & REF & \\
\hline Doping is mainly a problem of fair-play & & $1.32(0.12-4.98)$ & $1.69(0.11-5.87)$ & REF \\
\hline DOPING IN KICKBOXING QSU $¥$ & 0.30 & & & \\
\hline I don't think that doping occurs & & REF & REF & \\
\hline Not sure of doping in kickboxing & & $10.00(0.92-34.21)$ & $1.67(0.32-2.68)$ & \\
\hline Occurs, but rarely & & $2.51(0.63-9.83)$ & $1.71(0.40-6.87)$ & \\
\hline Doping is common & & $3.55(1.25-4.11)^{*}$ & $5.00(1.43-17.57) *$ & REF \\
\hline \multicolumn{5}{|c|}{ AOR - Adjusted Odds Ratio, 95\%CI - 95\% confidence interval; } \\
\hline \multicolumn{5}{|c|}{ QSU presents variables derived from the Questionnaire of Substance Use; } \\
\hline \multicolumn{5}{|c|}{ KD presents variables derived from the Knowledge on Doping Questionnaire; } \\
\hline \multicolumn{5}{|c|}{${ }^{K S N}$ presents variables derived from the Knowledge on Sport Nutrition Questionnaire; } \\
\hline \multicolumn{5}{|c|}{ CTEOSQ presents variables derived from the Croatian Version of the Task and Ego Orientation } \\
\hline \multicolumn{5}{|c|}{ in Sport Questionnaire; ${ }^{*}$ statistically significant ORs at $p<0.05$} \\
\hline \multicolumn{5}{|c|}{${ }^{¥}$ crude Odds Ratio presented for this variable } \\
\hline
\end{tabular}


Female athletes were better educated and achieved higher competitive results than their male peers. All tested athletes consumed dietary supplements at least irregularly. The 28 athletes $(21.53 \%)$ declared potential doping behavior, 42 athletes $(32.3 \%)$ were not sure about doping behavior in future, and 60 athletes (46.2\%) reported no tendency toward doping behavior in future. More than $50 \%$ of athletes were of the opinion that doping was primarily a "problem of fair play" (Table 2).

The personal opinion about high prevalence of doping in kickboxing was found to be strongly associated with "potential doping behavior" for those athletes who declared a positive tendency $(\mathrm{OR}=3.55 ; 95 \% \mathrm{CI}=1.25-4.11 ; \mathrm{p}$ $<0.05$ ), and those who were uncertain about their future doping behavior $(\mathrm{OR}=5.00$; $95 \% \mathrm{CI}=1.43$ 17.57; $\mathrm{p}<0.05)$. The associations with potential doping behavior and other variables obtained by the QSU, KSN, KD and CTEOSQ were examined by multinomial logistic regression with "doping in kickboxing" as a confounding factor. Experience in kickboxing was associated with positive intention toward doping behavior in the future $(\mathrm{OR}=1.11 ; 95 \% \mathrm{CI}=1.00-1.23 ; \mathrm{p}<0.05)$. Positive intention toward doping behavior in the future was lower in those who possessed better knowledge on nutrition $(\mathrm{OR}=0.62 ; 95 \% \mathrm{CI}=0.33$ $0.98 ; \mathrm{p}<0.05$ ). The sporadic (irregular) consumption of dietary supplements was found in those kickboxers with "neutral" opinion about potential doping behavior in the future $(\mathrm{OR}=$ 4.27; $95 \% \mathrm{CI}=1.52-11.97 ; \mathrm{p}<0.05)$.

\section{Discussion}

With regard to study hypotheses, we may report similar tendencies toward potential doping behaviors in males and females. Second, personal opinion of the doping presence in kickboxing was positively, knowledge on sports nutrition was negatively, while task- and ego-motivation were not found as being significantly associated to potential doping behavior in kickboxers. Prior to discussing those findings, we will shortly overview the prevalence of doping behavior in kickboxing

Of the sports investigated to date by the QSU, the highest positive tendency toward doping was reported in rugby and racket sports (in both cases more than $50 \%$ declared no potential doping behavior in males) (Rodek et al., 2012; Sajber et al., 2013; Sekulic et al., 2014). Therefore, with only $46 \%$ kickboxers who reported a negative tendency toward personal doping behavior in the future, the likelihood of doping in kickboxing is actually alarming. This directly confirms the recent WADA findings where kickboxing is evidenced as a martial art with a highest proportion of adverse analytical findings (WADA, 2015), which asks for a serious intervention. Also, such high prevalence of potential doping behavior is in accordance with the opinion that high risk of doping behavior could be expected in sports with high risk of injury and where athletes are engaged in weightcutting (Babwah, 2014; Sekulic et al., 2014)

Previous studies that investigated athletes from the region (i.e. territory of former Yugoslavia) regularly evidenced male athletes as being more prone to doping than females (Rodek et al., 2012; Sajber et al., 2013). Therefore, our finding of a similar tendency toward doping in both genders is not in agreement with results reported so far. Kickboxing, similar to the majority of striking sports, has primarily been organized around the capabilities of the male body, and women's interests in this sport are often questioned (Mierzwinski et al., 2014; Spencer, 2014). In a recent study on women involved in a sport very similar to kickboxing (i.e., Mixed Martial Arts), investigators accurately depicted some of the known gender-specific issues, including gendered experiences of shame in relation to fighting, physical marks (i.e., injuries), and sexual intimacy (i.e., close physical contact of bodies) (Mierzwinski et al., 2014). Although they did not investigate doping issues specifically, we believe that their findings could easily be applied to doping behavior as well. In general, it is difficult for women involved in kickboxing to be viewed as "typical" in terms of their sport-related motivation as well as their sport-related behaviors (such as doping behavior). Taking this into consideration, the relatively high tendency toward doping in female kickboxers is not surprising.

Our finding that most of the studied kickboxers observe doping as (mainly) a problem of fair-play is inconsistent with previous findings from other sports (Kondric et al., 2013; Sajber et al., 2013). More precisely, studies conducted so far 
highlight that the majority of athletes are primarily concerned about the health-related side effects of doping (Kondric et al., 2013; Sajber et al., 2013). It is probable that athletes involved in kickboxing are aware that participating in kickboxing is a health-risk behavior in and of itself. Consequently, athletes' perceptions of the health risks associated with doping may be moderated by the constant threat of being (seriously) injured in training and in competition (Lystad, 2015; Tanriverdi et al., 2007; Zazryn et al., 2003). Therefore, when compared with their everyday risk of injury, the health-related side effects of doping may not seem as serious.

A more positive doping tendency was found in athletes who perceived that doping was prevalent in kickboxing, and this is consistent with previous studies (Kondric et al., 2011; Rodek et al., 2013). This relationship can be largely explained by the social psychological theory of self-categorization (Turner and Oakes, 1986). According to this theory, people adopt the norms, behaviors and beliefs of their peers, i.e., members of the same group (i.e. sports community). Athletes who believe that doping is present in kickboxing (either because they are personally aware of it from knowing individuals who use doping, or because they purely suspect that doping occurs) may not believe that they will be able to achieve the results they are striving for without doping.

This is not the first time that sports nutrition knowledge has been found to be protective against potential doping behavior; such results have been reported previously in international-level tennis players (Kondric et al., 2013). Although we are not able to provide a clear explanation of this relationship, we can highlight one reason that should be investigated more closely in future studies. Greater knowledge of sports nutrition (i.e., a higher KSN score) could in fact mean that an athlete eats properly and combines his or her diet with the necessary dietary supplementation. As a result, their working capacity would be enhanced, and doping behavior would be less probable.

The motivation to participate in sport is an important factor of sport commitment, and studies performed so far have found certain relationships between motivational factors and doping attitudes (Allen et al., 2015; Sas-
Nowosielski and Swiatkowska, 2008; Zucchetti et al., 2015). Therefore, we initially hypothesized that task- and ego-motivation would be specifically associated to doping behavior even in kickboxing. However, herein we found no significant relationship between motivational variables and doping behavior. Therefore, our results are in certain disagreement with previous reports that found significant associations between different motivational factors and attitudes toward performance-enhancement drugs and doping (Allen et al., 2015; Sas-Nowosielski and Swiatkowska, 2008; Zucchetti et al., 2015). There are several probable explanations for the lack of the relationship between task- and egomotivation and potential doping behavior in kickboxing athletes.

First, it might be explained by the differences between our studied sample of participants, and those athletes observed in previous studies. Namely, previous studies sampled athletes involved in different sports, and/or athletes who were highly variable in their sport-achievement (Allen et al., 2015; SasNowosielski and Swiatkowska, 2008; Zucchetti et al., 2015). Meanwhile, we studied athletes from only one sport, who all achieved high-competitive results. Consequently, it is probable that the general variance of the motivational factors for the studied kickboxers is truncated, which did not allow identification of the statistically significant level of the association between observed variables (Huck, 2012). As a certain support to this observation, we may indicate that the variance (standard deviation) of the task- and egoorientation in kickboxers is evidently lower $(\mathrm{SD} /$ Mean $=14 \%$ and $11 \%$ for task and egoorientation for male kickboxers, respectively) than among athletes studied previously by the same questionnaire $(\mathrm{SD} / \mathrm{Mean}=14-16 \%$ and $32-35 \%$ for task and ego-orientation for team-sport athletes, respectively) (Baric and Bucik, 2009; Baric and Horga, 2007). Some specific issues related to taskand ego-orientation in kickboxing are discussed in the following paragraph.

It is generally accepted that the egooriented athlete tends to assess the level of his/her competence with reference to performance of others. More precisely, athletes with high egoorientation experience success only when his/her performance is better compared to others (Baric 
and Horga, 2007). However, kickboxing is a martial art. Therefore, kickboxing athletes are specifically ego-oriented, since their personal sport achievement is directly evidenced in "being better" than the opponent. One can argue that this is the case in all sports, yet in kickboxing this is highly emphasized. Namely, in this sport the final achievement is evidenced as "true" destruction of the opponent that frequently results even in serious injuries (Tanriverdi et al., 2007). Therefore, when compared to athletes studied by the same questionnaire, ego-orientation of athletes studied herein was relatively high $(2.99 \pm 0.91 ; 2.64 \pm 0.91$; $5.71 \pm 0.65$ for male soccer players, handball players and kickboxers, respectively) (Baric and Horga, 2007).

With regard to the lack of a relationship between task-orientation and potential doping behavior, another characteristic of kickboxing should be briefly explained. During the first phases of involvement in this sport, athletes are almost exclusively committed to the mastery of kickboxing techniques (i.e. there are no organized competitions). Therefore, in the early stages of practicing kickboxing, there is actually no "normative-based criteria", as the mastery of the sport-skills is an absolute prerequisite for competition (Buse and Santana, 2008). Consequently, athletes are at that phase of sport development almost exclusively oriented to demonstration of mastery, which is directly related to task-orientation (Baric and Bucik, 2009; Baric and Horga, 2007). It is unlikely that people with low task-orientation will persist in kickboxing training throughout the first phases of sport participation. Finally, task-orientation of those kickboxing athletes who achieved high competitive result is expected to be high. This is indirectly confirmed by our results since taskorientation reported for our studied kickboxers is evidently higher than task-orientation reported previously when athletes involved in other sports were studied by the CTEOSQ $(4.13 \pm 0.69 ; 4.20 \pm$ $0.70,6.74 \pm 0.94$ for handball, soccer and kickboxing, respectively) (Baric and Horga, 2007).

The main study limitation is the crosssectional study design. Accordingly, the results of the statistical analyses indicate a relationship, but causality cannot be determined. Additionally, the number of male athletes was three times greater than that of female athletes. However, taking into consideration the performance level and the number of studied athletes, we believe that our findings could contribute to the knowledge in this field.

\section{Conclusion}

Sports officials should be directly informed about the findings on high risk for doping behavior in kickboxing. Since there were no gender difference in the doping tendency, the high prevalence of potential doping behavior is particularly alarming for females. Although most anti-doping campaigns highlight the health hazards of doping behavior, our findings indicate the need for a somewhat different approach to anti-doping efforts for kickboxing, i.e., one in which issues of fair play should be the primary emphasis.

A higher positive tendency toward doping behavior in the future was found in more experienced athletes and athletes who were convinced that doping was prevalent in kickboxing. A lower level of potential doping behavior was found in athletes who had greater knowledge of sports nutrition; therefore, the improvement of knowledge in sports nutrition should be considered as an important component of anti-doping efforts in this sport.

In the sample of high-level kickboxers, task- and ego-orientation were not found to be related to doping behavior. It is probable that the high-competitive level and age of the athletes observed herein did not allow to define the relationship between these variables and doping behavior. Therefore, future studies should explore the problem while studying other sports and younger athletes. 


\section{References}

Allen J, Taylor J, Dimeo P, Dixon S, Robinson L. Predicting elite Scottish athletes' attitudes towards doping: examining the contribution of achievement goals and motivational climate. J Sport Sci, 2012; 33: 899906

Babwah T. Pain, injury and related behaviours among footballers partaking in tournaments. Res Sports Med, 2015; 22: 334-345

Backhouse SH, Whitaker L, Petroczi A. Gateway to doping? Supplement use in the context of preferred competitive situations, doping attitude, beliefs, and norms. Scand J Med Sci Sports, 2013; 23: 244-252

Baric R, Bucik V. Motivational differences in athletes trained by coaches of different motivational and leadership profiles. Kinesiology, 2009; 41: 181-194

Baric R, Horga S. Psychometric properties of the Croatian version of task and ego orientation in sport questionnaire (CTEOSQ). Kinesiology, 2007; 38: 135-142

Buse GJ, Santana JC. Conditioning strategies for competitive kickboxing. Strength Cond J, 2008; 30: 42-48

Franchini E, Brito CJ, Artioli GG. Weight loss in combat sports: physiological, psychological and performance effects. J Int Soc Sports Nutr, 2012; 9. doi: Artn 52 10.1186/1550-2783-9-52

Huck SW. Reading Statistics and Research: Pearson; 2012

Kondric M, Sekulic D, Petroczi A, Ostojic L, Rodek J, Ostojic Z. Is there a danger for myopia in anti-doping education? Comparative analysis of substance use and misuse in Olympic racket sports calls for a broader approach. Subst Abuse Treat Prev Policy, 2011; 6: 27

Kondric M, Sekulic D, Uljevic O, Gabrilo G, Zvan M. Sport nutrition and doping in tennis: an analysis of athletes' attitudes and knowledge. J Sports Sci Med, 2013; 12: 290-297

Lystad RP. Injuries to professional and amateur kickboxing contestants: A 15-Year Retrospective Cohort Study. Orthop J Sports Med, 2015; 3: 2325967115612416

Mierzwinski M, Velija P, Malcolm D. Women's experiences in the mixed martial arts: a quest for excitement? Sociol Sport J, 2014; 31: 66-84

Nassib S, Nassib SH, Hachana Y, El Kefi R, Souissi N. Muscle and anthropometric profile of the athletes of the Tunisian national team of senior male kickboxing during pre-competitive. Fundam Clin Pharmacol, 2011; 25: 106-106

Pettersson S, Ekstrom MP, Berg CM. Practices of weight regulation among elite athletes in combat sports: a matter of mental advantage? J Athl Train, 2013; 48: 99-108

Rodek J, Idrizovic K, Zenic N, Perasovic B, Kondric M. Differential analysis of the doping behaviour templates in three types of sports. Coll Antropol, 2013; 37: 211-217

Rodek J, Sekulic D, Kondric M. Dietary supplementation and doping-related factors in high-level sailing. J Int Soc Sports Nutr, 2012; 9: 51

Sajber D, Rodek J, Escalante Y, Olujic D, Sekulic D. Sport nutrition and doping factors in swimming; parallel analysis among athletes and coaches. Coll Antropol, 2013; 37S2: 179-186

Sas-Nowosielski K, Swiatkowska L. Goal orientations and attitudes toward doping. Int J Sports Med, 2008; 29: 607-612

Sekulic D, Bjelanovic L, Pehar M, Pelivan K, Zenic N. Substance use and misuse and potential doping behaviour in rugby union players. Res Sports Med, 2014; 22: 226-239

Sekulic D, Kostic R, Miletic D. Substance use in dance sport. Med Probl Perf Art, 2008; 23: 66-73

Sekulic D, Tahiraj E, Zvan M, Zenic N, Uljevic O, Lesnik B. Doping Attitudes and Covariates of Potential Doping Behaviour in High-Level Team-Sport Athletes; Gender Specific Analysis. J Sports Sci Med, 2016; 15: 606-615 
Spencer DC. 'Eating clean' for a violent body: mixed martial arts, diet and masculinities. Womens Stud Int Forum, 2014; 44: 247-254

Tanriverdi F, Unluhizarci K, Coksevim B, Selcuklu A, Casanueva FF, Kelestimur F. Kickboxing sport as a new cause of traumatic brain injury-mediated hypopituitarism. Clin Endocrinol (Oxf), 2007; 66: 360-366

Turner JC, Oakes PJ. The significance of the social identity concept for social-psychology with reference to individualism, interactionism and social-influence. Br J Soc Psychol, 1986; 25: 237-252

WADA; https://wada-main-prod.s3.amazonaws.com/wada_2014_anti-doping-testing-figures_fullreport_en.pdf.; accessed April 142016

Zazryn TR, Finch CF, McCrory P. A 16 year study of injuries to professional kickboxers in the state of Victoria, Australia. Br J Sports Med, 2003; 37: 448-451

Zucchetti G, Candela F, Villosio C. Psychological and social correlates of doping attitudes among Italian athletes. Int J Drug Policy, 2015; 26: 162-168

\section{Corresponding author:}

\section{Damir Sekulic}

University of Split, Faculty of Kinesiology

Teslina 6, Split - 21000, Croatia

Phone: +38521302440

Fax: +38521302440

E-mail: dado@kifst.hr 